\title{
Nitric Oxide (NO) and Arginine as Factors for Increasing Poultry Meat Productivity
}

\section{Vladimir Titov ${ }^{1,2}$, Anna Dolgorukova ${ }^{1}$, Lyudmila Khasanova ${ }^{1}$, Ivan Kochish ${ }^{2}$, and Maxim Korenyuga ${ }^{2}$}

${ }^{1}$ Federal Scientific Center All-Russian Research and Technological Poultry Institute of the Russian Academy of Sciences, Moscow Region, Sergiev Posad, Russia

${ }^{2}$ Moscow State Academy of Veterinary Medicine and Biotechnology - MVA named after K.I. Skryabin, Moscow, Russia

ORCID:

Vladimir Titov: http://orcid.org/0000-0002-2639-7435

\section{Abstract}

Nitric oxide (NO) is intensively synthesized in the embryo of birds. There is evidence that NO mediates myogenesis at the embryonic stage. In this regard, it might be possible to control muscle development and meat productivity by modulating NO synthesis. Using high-precision and a highly sensitive enzymatic sensor, the authors

Corresponding Author:

Vladimir Titov

vtitov43@yandex.ru

Published: 5 April 2021

Publishing services provided by

Knowledge E

(c) Vladimir Titov et al. This article is distributed under the terms of the Creative Commons Attribution License, which permits unrestricted use and redistribution provided that the original author and source are credited.

Selection and Peer-review under the responsibility of the DonAgro Conference Committee. found that it is not the rate of synthesis that correlates with meat productivity, but the rate of NO oxidation to nitrate, which occurs in the tissues of the embryo. In broilers, it is several orders of magnitude higher than in layers. This indicator is solely due to the characteristics of embryonic tissues and is allelically determined. In-ovo arginine supplementation, a source of NO, did not lead to a significant increase in its synthesis and oxidation, but occasionally increased live weight gain, which was likely associated with a deficiency of free arginine and was not directly related to the effect of nitric oxide. Exogenous NO donors were oxidized with the same intensity as endogenous donors. These compounds did not have a significant effect on growth rate. Also, a reduction of $50 \%$ in the rate of NO synthesis under the action of a NO synthase inhibitor did not affect this parameter. Thus, regulation of poultry meat productivity is possible by modulating gene expression related to embryonic NO oxidation and ensuring an optimal amino acid and energy equilibrium, rather than by promoting embryonic NO synthesis.

Keywords: Nitric oxide (NO), NO donor compounds, nitrate, arginine, live weight

\section{Introduction}

Embryogenesis is accompanied by intensive synthesis of nitric oxide (NO). This is a universal cellular mediator of many physiological functions: smooth muscle tone, apoptosis and cell differentiation, lactation, platelet aggregation [1-3]. Being able to regulate these processes by means of NO could provide overwhelming possibilities to control a physiological status of the body and productivity of farm animals. However, 
this is hampered by the lack of ideas of the mechanism of NO effects. This, in turn, is caused by insufficient methods to control composition of NO metabolites in living tissues. Currently, NO is known to interact with physiological targets through the socalled compounds - depots or compounds - donors: dinitrosyl-iron complexes (DNIC), as well as S-nitrosothiols (RSNO) and high-molecular-weight nitrates that can transform into DNIC $\left(\mathrm{RNO}_{2}\right)[1,4,5]$. A synthesized $\mathrm{NO}$ molecule is likely to be immediately incorporated into these compounds [1]. In this case, two indicators can be informative: a total of NO metabolites, as an indicator of synthesis rate, and a ratio of NO donor compounds with end products of NO oxidation, nitrate and nitrite, as an indicator of NO metabolism rate. Nitrite is normally found in trace amounts in most tissues [6, 7].

Due to the lack of prompt determination of all NO donor compounds, NO effects were judged by the effects of NO synthase inhibitors [8-11], the effects of arginine supplementation, a source of $\mathrm{NO}$ [8, 9], as well as NO donor compounds [8, 12]. However, to establish the role of $\mathrm{NO}$ in specific processes, information is needed on the features of NO synthesis and metabolism and their relationship with the process concerned.

According to some researchers, nitric oxide mediates the process of myogenesis. These conclusions were drawn based on the use of NO synthase inhibitors, an enzyme that catalyzes NO synthesis from arginine [8-11], as well as the use of compounds NO donors [8, 12] and arginine - a source of NO [8, 9, 13]. However, cellular-level patterns are not always reproduced at the organismic level $[8,13]$. Thanks to the use of highly sensitive and highly specific enzymatic sensor, nitric oxide was found to accumulate in avian embryos as part of NO donor compounds. The rate of NO synthesis is approximately the same in all embryos of birds of one species. However, the rate of $\mathrm{NO}$ oxidation to nitrate is different for layers and broilers. Having explored the embryos of over 40 breeds, lines and crosses of 5 bird species, the authors found that the intensity of $\mathrm{NO}$ embryonic oxidation in broilers is many times higher than in layers $[6,7]$. Intense NO synthesis and oxidation was found to be present in the embryo. In the body of hatched chickens, these indicators decrease and level out. It is the growth rate of chickens, though, that correlates with the intensity of NO embryonic oxidation $[6,7]$. Moreover, oxidation mainly occurs in skeletal muscles [7]. Breeding to increase meat productivity is always accompanied by the promotion of embryonic NO oxidation $[6,7]$. A number of questions come up as to the mechanism of this relationship and the likelihood to control the development of the bird's body by modulating embryonic NO synthesis.

The paper aims to find out the possibility of modulating the growth rate of birds by regulating the intensities of NO synthesis and oxidation in avian embryos 
TABLE 1: Concentration of NO donors and nitrate in Hisex White and Ross 308 embryos on days 7 and 14 of incubation. Effect of arginine (A) and nitro-L-arginine (L-NA) ***

\begin{tabular}{|c|c|c|c|c|c|}
\hline \multirow[t]{2}{*}{ No. } & \multirow[t]{2}{*}{ Object } & \multicolumn{2}{|c|}{ Day 7} & \multicolumn{2}{|c|}{ Day 14} \\
\hline & & $\begin{array}{c}\text { NO donors, } \\
\mu \mathrm{M}\end{array}$ & Nitrate, $\mu \mathrm{M}$ & $\begin{array}{c}\text { NO donors, } \\
\mu \mathrm{M}\end{array}$ & Nitrate, $\mu \mathrm{M}$ \\
\hline 1. & Hisex White (control) & $133.9 \pm 7.1$ & $<0.1$ & $639.6 \pm 18.3$ & $2.0 \pm 1.2$ \\
\hline 2. & +0.3 $\mathrm{ml}$ saline prior to incubation & $131.4 \pm 7.4$ & $<0.1$ & $640.8 \pm 19.1$ & $2.2 \pm 1.2$ \\
\hline 3. & $+0.3 \mathrm{ml}$ saline on day 11 of incubation & $136.5 \pm 7.9$ & $<0.1$ & $651.8 \pm 19.7$ & $2.6 \pm 1.3$ \\
\hline 4. & $\begin{array}{l}+100 \mu \mathrm{M} \text { DNIC/GSH prior to } \\
\text { incubation }\end{array}$ & $212.2 \pm 8.1$ & $<0.1$ & - & - \\
\hline 5. & $+100 \mu \mathrm{M}$ L-HA prior to incubation & $39.5 \pm 3.1$ & $<0.1$ & $638.2 \pm 19.3$ & $1.8 \pm 1.3$ \\
\hline 6. & $+100 \mu \mathrm{M}$ L-HA on day 11 of incubation & $134.8 \pm 6.9$ & $<0.1$ & $375.6 \pm 12.3$ & $2.0 \pm 1.4$ \\
\hline 7. & $\begin{array}{l}\text { +100 } \mu \mathrm{M} \text { L-HA + } 500 \mu \mathrm{M} \text { A prior to } \\
\text { Incubation }\end{array}$ & $135.7 \pm 7.1$ & $<0.1$ & $642.1 \pm 18.9$ & $1.5 \pm 1.0$ \\
\hline 8. & $+500 \mu \mathrm{M}$ A prior to incubation & $136.2 \pm 7.3$ & $<0.1$ & - & - \\
\hline 9. & Ross 308 (control) & $2.1 \pm 0.9$ & $142.1 \pm 7.7$ & $13.1 \pm 2.2$ & $759.4 \pm 17.2$ \\
\hline 10. & $+0.3 \mathrm{ml}$ saline prior to incubation & $2.9 \pm 1.0$ & $136.9 \pm 7.8$ & $11.4 \pm 2.1$ & $751.4 \pm 20.0$ \\
\hline 11. & +0.3 $\mathrm{ml}$ saline on day 11 of incubation & $3.1 \pm 1.0$ & $143.9 \pm 8.1$ & $12.1 \pm 2.6$ & $761.3 \pm 20.4$ \\
\hline 12. & $\begin{array}{l}+100 \mu \mathrm{M} \text { DNIC/GSH prior to } \\
\text { incubation }\end{array}$ & $30.1 \pm 3.5$ & $201.4 \pm 8.4$ & - & - \\
\hline 13. & $+100 \mu \mathrm{M}$ HA prior to incubation & $1.5 \pm 0.8$ & $37.9 \pm 2.9$ & $12.1 \pm 1.9$ & $746.3 \pm 17.1$ \\
\hline 14. & +100 $\mu \mathrm{M}$ L-HA on day 11 of incubation & $2.8 \pm 1.0$ & $139.5 \pm 7.4$ & $7.2 \pm 1.1$ & $472.6 \pm 11.8$ \\
\hline 15. & $\begin{array}{l}\text { +100 } \mu \mathrm{M} \text { L-HA + } 500 \mu \mathrm{M} \text { A prior to } \\
\text { incubation }\end{array}$ & $2.7 \pm 0.9$ & $143.3 \pm 8.1$ & - & - \\
\hline 16. & $+500 \mu \mathrm{M}$ A prior to incubation & $3.0 \pm 0.9$ & $142.9 \pm 7.9$ & - & - \\
\hline 17. & $+1500 \mu \mathrm{M}$ A on day 11 of incubation & $2.8 \pm 1.1$ & $140.3 \pm 8.1$ & $11.5 \pm 1.9$ & $764.7 \pm 19.9$ \\
\hline
\end{tabular}

* Calculated concentrations of L-HA, A and DNIC/GSH in eggs is given, based on egg size and concentrations of administered solution.

** The concentration of $\mathrm{NO}_{2}{ }^{-}+\mathrm{RNNO}$ in all samples was $<0.1 \mu \mathrm{M}$.

\section{Methods and Equipment}

Fertilized eggs of the two chicken crosses (Hisex White and Ross 308; Genofond) were used. The embryos were homogenized on day 7 in a glass homogenizer ( 8 min at 40 frictions per min and $6{ }^{\circ} \mathrm{C}$ ). The embryos were homogenized on day 14 with a tissue grinder [6, 7].

The amino acid composition of the embryo was determined via ion-exchange chromatography with post-column derivatization with ninhydrin reagent and subsequent detection at wavelengths of 440 and $570 \mathrm{~nm}$. HPLC system Sykam (Germany).

The content of NO metabolites was determined using the enzymatic sensor based on the ability of nitrite $\left(\mathrm{NO}_{2}{ }^{-}\right)$, nitrosoamines (RNNO), nitrosothiols (RSNO), dinitrosyl-iron complexes (DNIC) and nitro derivatives of high-molecular-weight compounds $\left(\mathrm{RNO}_{2}\right)$ to inhibit catalase in the presence of haloid ions. The ability to inhibit is lost under 
TABLE 2: Effect of L-NA and arginine (A) on live weight gain of layer cross (Hisex White) and broiler cross (Ross 308) *

\begin{tabular}{|c|c|c|c|c|c|}
\hline \multirow[t]{2}{*}{ No. } & \multirow[t]{2}{*}{ Object } & \multirow[t]{2}{*}{ Egg weight, g } & \multicolumn{3}{|c|}{ Body weight, g } \\
\hline & & & Day 1 & Day 7 & Day 14 \\
\hline 1. & Hisex White (control) & $62.1 \pm 0.3$ & $39.9 \pm 0.5$ & $59.3 \pm 1.2$ & $114,1 \pm 3,2$ \\
\hline 2. & $+0.3 \mathrm{ml}$ saline prior to incubation & $62.3 \pm 0.2$ & $40.2 \pm 0.5$ & $58.2 \pm 1.2$ & $113,2 \pm 3,3$ \\
\hline 3. & $+0.3 \mathrm{ml}$ saline on day 11 of incubation & $62.4 \pm 0.2$ & $40.1 \pm 0.5$ & $57.9 \pm 1.2$ & $112.2 \pm 3.3$ \\
\hline 4. & $\begin{array}{l}\text { +100 } \mu \mathrm{M} \text { DNIC/GSH prior to } \\
\text { incubation }\end{array}$ & $62.7 \pm 0.3$ & $40.1 \pm 0.5$ & $57.9 \pm 1.1$ & $114.3 \pm 3.1$ \\
\hline 5. & $+100 \mu \mathrm{M}$ L-HA prior to incubation & $62.3 \pm 0.3$ & $41.0 \pm 0.5$ & $67.5 \pm 1.1$ & $121.9 \pm 2.8$ \\
\hline 6. & $+100 \mu \mathrm{M}$ L-HA on day 11 of incubation & $62.4 \pm 0.3$ & $41.1 \pm 0.6$ & $59.4 \pm 1.1$ & $102.6 \pm 2.8$ \\
\hline 7. & Ross 308 & $67.3 \pm 0.7$ & $47.1 \pm 0.5$ & $180.9 \pm 2.8$ & $486.9 \pm 7.5$ \\
\hline 8. & +0.3 $\mathrm{ml}$ saline prior to incubation & $67.3 \pm 0.7$ & $47.6 \pm 0.5$ & $182.1 \pm 2.7$ & $490.5 \pm 7.4$ \\
\hline 9. & $+0.3 \mathrm{ml}$ saline on day 11 of incubation & $67.1 \pm 0.6$ & $46.8 \pm 0.5$ & $178.6 \pm 2.7$ & $481.8 \pm 7.7$ \\
\hline 10. & $\begin{array}{l}\text { +100 } \mu \mathrm{M} \text { DNIC/GSH prior to } \\
\text { incubation }\end{array}$ & $67.2 \pm 0.6$ & $47.7 \pm 0.5$ & $183.7 \pm 3.0$ & $491.8 \pm 7.2$ \\
\hline 11. & +100 $\mu \mathrm{M}$ L-HA prior to incubation & $67.6 \pm 0.4$ & $47.4 \pm 0.4$ & $192.1 \pm 2.3$ & $\begin{array}{c}518.9 \pm 8.3 \\
p<0.05\end{array}$ \\
\hline 12. & +100 $\mu \mathrm{M}$ HA until incubation day 11 & $65.3 \pm 0.7$ & $45.5 \pm 0.6$ & $178.8 \pm 2.9$ & $491.9 \pm 8.3$ \\
\hline 13. & +500 $\mu \mathrm{M}$ A prior to incubation & $67.9 \pm 0.5$ & $47.0 \pm 0.5$ & $193.5 \pm 2.9$ & $\begin{array}{c}509.7 \pm 7.6 \\
p<0.05\end{array}$ \\
\hline 14. & +1500 $\mu \mathrm{M} A$ on day 11 of incubation & $64.6 \pm 0.6$ & $46.7 \pm 0.7$ & $181.7 \pm 3.5$ & $476.6 \pm 7.8$ \\
\hline
\end{tabular}

* Calculated concentrations of L-HA, A and DNIC/GSH in eggs is given, based on egg size and concentrations of administered solution.

the influence of factors that are different for each group of compounds [6]. The nitrate content in the samples was estimated after its preliminary reduction with vanadium trichloride to nitrite with subsequent quantitative determination [6]. The method makes it possible to estimate the content of NO derivatives without pretreatment of samples with a sensitivity of up to $50 \mathrm{nM}$. The installation featured a Dithermanal analyzer (Hungary) [6].

The preparation of dinitrosyl-iron complex containing two molecules of thiol ligand glutathione (DNIC/GSH) was used as exogenous NO donor. It was prepared by the method described by A.F. Vanin. [1, 4]. N $\omega$ - nitro-L-arginine (L-NA) was used as an inhibitor of NO synthesis. The preparation of L- arginine (Sigma-Aldrich) was used as a source of NO. The solutions in sterile normal saline were injected in an air pocket in ovo prior to incubation and until incubation day 11. All solutions were injected into eggs in a dose of $0.3 \mathrm{ml}$

Stimul Ink-1000 incubators (Russia) were used for incubation. The temperature of incubation was $37.6{ }^{\circ} \mathrm{C}$ and $37.2{ }^{\circ} \mathrm{C}$ until hatch. The control and experimental groups contained 50 eggs each. Hatched chicks were kept in cells. The birds fed on feed stuff 


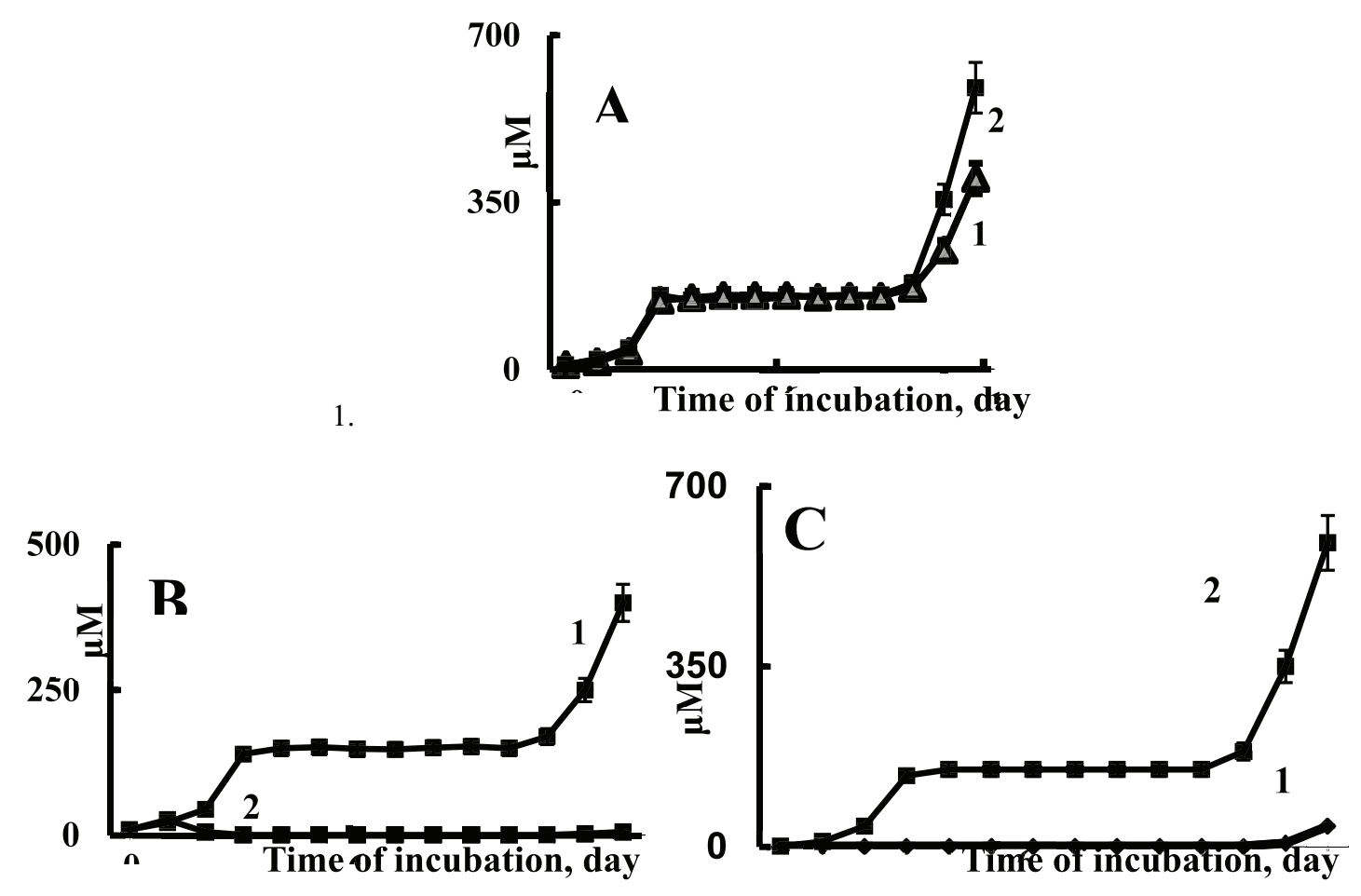

Figure 1: Total pool of all NO metabolites (A), NO donors (B), nitrate (C) in embryos of layer Hisex White (1) and broiler Ross 308 (2) vs time of incubation

PK5. Since the more the bird weight, the more the gutted carcass yield [14], live weight gain was used as a criterion for muscle tissue growth.

In the tables, the data are presented as means and standard errors of means $(M \pm m)$. The significance of differences between the compared indicators was determined using the Student's t-test. Differences were considered statistically significant at $p<0.05$.

\section{Results}

Our data suggest that initially fertilized chicken eggs, both broiler and layer, contains $42 \pm 4 \mathrm{mM}$ arginine. Of these, $680 \pm 30 \mu \mathrm{M}$ account for the unbound pool of free amino acids. These data agree with the literature [14]. Fertilized chicken eggs contain several $\mu \mathrm{M}$ nitro- and nitroso-compounds. They accumulate in the first three days of development. Basically, NO donor compounds accumulate: S-nitrosothiols (RSNO), various nitrosyl-iron complexes, high-molecular-weight nitrates $\left(\mathrm{RNO}_{2}\right)$. They mainly accumulate in layer embryos and are oxidized to nitrate in broiler embryos (Fig. 1b, c, Table 1 (1-3 and 9-11)). According to the data, NO donors accumulate in the amniotic fluid, and nitrate is actively transported beyond [7]. In total, about 130-140 $\mu \mathrm{M}$ of NO metabolites are accumulated by day 3 (Fig. 1a). This is a small amount relative to the total arginine 
content in eggs, but about a quarter of its free pool. Starting from day $11, \mathrm{NO}$ derivatives intensively accumulate in the embryo (Fig. 1a-c, Table 1 (1-3 and 9-11)), due to the stimulation of NO synthesis in embryo tissues [7]. By the end of the embryonic period, the concentration of NO derivatives can reach millimolar (Fig. 1a). Consequently, a significant amount of free arginine is required for NO synthesis. The efficiency of its release from the bound state can be of great importance, since there is barely any synthesis during the embryonic period [14].

Injection of NO synthase inhibitor L-nitroarginine (L-HA) prior to incubation decreased the total concentrations of NO donors and nitrate in embryos on day 7, but on day 14, the content of NO metabolites in the control and experimental groups leveled off. That is, after 11 days, L-HA no longer has an inhibitory effect on the accumulation of NO derivatives (Table 1 ( 5 and 13)). This may be caused by two things: either the L-HA pool runs down or it cannot penetrate to the sites of NO synthesis, which after 11 days are concentrated in the embryonic cells [7]. However, after the administration of L-HA on day 11, it has an inhibitory effect, as evidenced by decreased NO derivatives on day 14 , compared with the control (Table 1 (6 and 14)). Consequently, the pool of injected L-HA in the embryo runs down over time.

Arginine (A), administered together with L-HA, prevented the inhibitory effect of the latter (Table 1 (7 and 15)). However, administration of arginine, even at a dose equal to that of free arginine in embryos, does not seem to stimulate NO synthesis, which can be judged by no substantial changes in concentration of NO derivatives (Table 1 $(8,16$ and 17)). L-HA injection in a dose that reduces NO synthesis by $70 \%$ at the initial stage of incubation and by $40 \%$ after 11 days (Table $1(5,6,13,14)$ ) did not negatively affect postembryonic growth. Moreover, in some cases, the administration of L-NA, like arginine, accelerated postembryonic growth (Table $2(11,13))$.

Exogenous NO donors are oxidized with the same intensity as endogenous donors (Tables 1 (4 and 12)). It makes sense to inject these compounds into the embryo only prior to incubation, since they cannot pass through the amnion formed until incubation day 3 [7]. They did not have a significant effect on the rate of postembryonic growth (Tables 2 (4 and 10)).

\section{Discussion}

In the first three to four days of development, NO accumulates in the amniotic fluid in composition of donor compounds [7]. It is not known where NO is synthesized at this stage of embryogenesis. Perhaps in the amnion [15]. Starting from day $11, N O$ is 
intensively synthesized in the tissues of the embryo and accumulates in composition of the donor compounds. These compounds accumulate most intensively in the liver. However, oxidation to nitrate occurs mainly in muscle tissue, from which nitrate is actively transported outside the amniotic sac [7]. Since muscle mass prevails over the mass of other tissues, hence most of the NO deposited in tissues of the broiler embryo is oxidized (Fig. 1b, c, Table 1 (9-11)).

After addition of exogenous DNIC/GSH preparation into the embryo, it is intensively oxidized in Ross 308 embryo and practically not oxidized in Hisex White embryo (Table 1 (4 and 12)). Thus, the rate of NO oxidation in the avian embryo is determined by the characteristics of the tissues. There is practically no spontaneous oxidation. According to the data, the rate of NO oxidation in the embryo varies by no more than $10 \%$ within the breed, line, and cross and does not depend on the sex of the embryo and the state of a laying hen. It also does not depend on incubation conditions [7]. Apparently, it is determined allelically.

But what is the mechanism of the relationship between myogenesis and NO oxidation? First of all, it is obvious that this oxidation is not necessary for myogenesis as such. In layer cross, it runs on almost with no oxidation (Fig. 1b, c, Table 1 (1-3)) and at the same rate as in broiler cross. No qualitative histological differences were found [7]. It is also obvious that such a high concentration of NO donors, which occurs in layer embryos, is not vital, since in broiler embryos these compounds are almost completely oxidized to nitrate (Fig. 1b, c, Table 1 (9-11)). The reduced content of NO donors under the action of NO synthase inhibitor L-HA either did not negatively affect the postembryonic growth of chicks (Tables 1, 2).

Then, what is the physiological significance of NO accumulation in the embryo? It is also found to be present in other animals [7]. It can be assumed that $\mathrm{NO}$ oxidation as such somehow initiates the development of muscle tissue. The data suggest that the amount of oxidized NO, at least its decrease and increase by several times, does not significantly affect the growth rate (Table 1, 2).

Hence, it is not NO oxidation that initiates muscle growth, but allelically determined features related to NO oxidation. The role of oxidation is not yet known.

Provided that the rate of embryonic NO oxidation is determined allelically, then it can be regulated by changing the expression of the corresponding genes. This appears to be the case while using white and green light during incubation. This is a well-known way to stimulate the growth rate $[16,17]$. According to the data, the use of light during incubation promoted NO oxidation in the embryo, but did not stimulate its synthesis. Thus, in layer embryos, up to $65 \%$ of NO donors are oxidized under the action of light, 
while in the control their oxidation is insignificant. This was accompanied by significant growth rate of $3-5 \%$ after hatch [7]

\section{Conclusion}

Based on the findings, a several-fold change in the intensity of NO synthesis in the embryo of chickens, as well as the exogenous administration of donor compounds did not significantly affect the growth rate of chicks.

In ovo addition of arginine, a source of nitric oxide, at a dose equal to that of free arginine already present in eggs, did not significantly affect the rate of NO synthesis and the rate of its oxidation to nitrate, which is determined solely by allelically determined features of embryonic tissues. However, NO synthesis requires arginine in an amount comparable to its free initial amount present in eggs. Perhaps that is why in ovo administration of arginine can have an effect on body weight gains. It is not directly related to NO synthesis and oxidation.

The most effective way to control the development of the bird's body, mediating nitric oxide, is to influence the number or status of physiological targets of NO, that is, the expression of the corresponding genes, provided that the amino acid and energy equilibrium is ensured.

\section{Funding}

\section{Acknowledgement}

The reported study was funded by RFBR, project number 20-016-00204-a

\section{Conflict of Interest}

The authors have no conflict of interest to declare.

\section{References}

[1] Vanin, A., Borodulin, R. and Mikoyan, V. (2017). Dinitrosyl Iron Complexes with Natural Thiol-Containing Ligands in Aqueous Solutions: Synthesis and Some PhysicoChemical Characteristics (A Methodological Review). Nitric Oxide, vol. 66, pp. 1-9. 
[2] Kim, Y., et al. (2000) Cellular Non-Heme Iron Content is a Determinant of Nitric Oxide-Mediated Apoptosis, Necrosis, and Caspase Inhibition. Journal of Biological Chemistry., vol. 275, pp. 10954-10961.

[3] Li, J., et al. (1997). Nitric Oxide Reversibly Inhibits Seven Members of the Caspase Family Via S-Nitrosylation. Biochemical and Biophysical Research Communications, vol. 240, pp. 419-424.

[4] Vanin, A. (2016). Dinitrosyl Iron Complexes with Thiol-Containing Ligands as a "Working Form" of Endogenous Nitric Oxide. Nitric Oxide, vol. 54, pp. 15-29.

[5] Lima, E., et al. (2005). Nitrated Lipids Decompose to Nitric Oxide and Lipid Radicals and Cause Vasorelaxation. Free Radical Biology and Medicine, vol. 39, pp. 532-539.

[6] Titov, V., et al. (2012) Nitric Oxide (NO) in Bird Embryogenesis: Physiological Role and Ability of Practical Use. World's Poultry Science Journal, vol. 68, pp. 83-95.

[7] Titov, V., et al. (2018) The Role of Nitric Oxide (NO) in the Body Growth Rate of Birds. World Poultry Science Journal, vol. 74, pp. 675-686.

[8] Cazzato, D., et al. (2014) Nitric Oxide Drives Embryonic Myogenesis in Chicken Through the Upregulation of Myogenic Differentiation Factors. Experimental Cell Research, vol. 320, pp. 269-280.

[9] Long, J., et al. (2006). Arginine Supplementation Induces Myoblast Fusion Via Augmentation of Nitric Oxide Production. Journal of Muscle Research and Cell Motility, vol. 27, pp. 577-584.

[10] Anderson, J. (2000). A Role for Nitric Oxide in Muscle Repair: Nitric Oxide-mediated Activation of Muscle Satellite Cells. Molecular Biology of the Cell, vol. 11, pp. 18591874.

[11] Stamler, J. and Meissner, G. (2001). Physiology of Nitric Oxide in Skeletal Muscle. Physiological Reviews, vol. 81, pp. 209-237.

[12] Ulibarri, J., et al. (1999). Nitric Oxide Donors, Sodium Nitroprusside and S-NitrosoN-Acetylpencillamine, Stimulate Myoblast Proliferation in Vitro. In Vitro Cellular \& Developmental Biolody - Animal, vol. 35, pp. 215-218.

[13] Li, Y., et al. (2016). In Ovo L-Arginine Supplementation Stimulates Myoblast Differentiation but Negatively Affects Muscle Development of Broiler Chicken after Hatching. Journal of Animal Physiology and Animal Nutrition, vol. 100, pp. 167-177.

[14] Fisinin, V., Zhuravlev, I. and Aydinyan, T. (1990). Embryonic Development of Poultry. Moscow: Agropromizdat.

[15] Khan, H., et al. (2012). Expression and Localization of NO Synthase Isoenzymes (iNOS and eNOS) in Development of the Rabbit Placenta. Journal of Reproduction and Development., vol. 58, pp. 231-236. 
[16] Rozenboin, l., et al. (2013) The Effect of Monochromatic Photostimulation on Growth and Development of Broiler Birds. General and Comparative Endocrinology, vol. 190, pp. 214-219.

[17] Sobolewska, A., et al. (2011) Myogenesis-Possibilities of its Stimulation in Chickens. Folia Biologica (Krakow), vol. 59, pp. 85-90. 